\title{
Table des Matières
}

\section{CINQUIEME PERIODE: $1600-1200$ \\ REACTION EGYPTIENNE CONTRE L'INVASION HYKSOSS. EXPANSION MILITAIRE DES PHARAONS EN ASIE}

\section{A. L'EMPIRE EGYPTIEN D'ORIENT}

I. Naissance de l'impérialisme Égyptien. Conquête de la Syrie

1. L'Egypte, puissance impériale . . . . . . . . . . .

a. Naissance de l'impérialisme égyptien, 29. - b. Syrie-Palestine, zone de protection pour l'Egypte, 30. - c. Conquête de la Nubie (15751556), 31.

2. Conquête du Harou (Palestine, Liban, Syrie) . . . . . .

a. Conquête de la Palestine, 33. - b. Conquête de la Phénicie, 34. - c. Conquête de la Syrie-Nord, 34. - d. L'Euphrate, frontière de l'Egypte, 35.

3. Entente égypto-mitannienne . . . . . . . . . . .

a. Réveil des Hittites et des Etats du Nord, 36. - b. Pacte d'alliance égypto-mitannien (vers 1420), 36. - c. Rapports avec Hittites, Assyriens, Kassites, 37.

II. L'EMPIRE ÉGYPTIEN D'ORIENT ET SES VOISINS DU NORD . . . 38

1. L'empire égyptien d'Orient . . . . . . . . . . . . . . .

a. Etendue et richesse des provinces orientales, 38. - b. Organisation politique des provinces orientales, 39. - c. Les tendances régionalistes encouragées et maintenues, 39. - d. Attributions des roitelets locaux, 40. - e. Protectorat libéral, 41. - f. Domination égyptienne et culture babylonienne, 41.

2. Rapports de l'Egypte et de la Crète . . . . . . . . . 42

3. L'Egypte, centre de la vie mondiale . . . . . . . . . 43

a. Centre principal du commerce international, 43. - b. Les bienfaits de la paix égyptienne, 44.

III. RÉvolution RELIGIEUSE EN EgYPTe: LE MONOTHÉISME SOlAIRE 46

1. Aton-Soleil, dieu unique, remplace Amon . . . . . . . 46

a. Objectif de la réforme religieuse, 46. - b. Amon, dieu dynastique et national, 47. - c. Aton, dieu unique et universel, 47.

2. Le monothéisme atonien ou solaire, produit de l'impérialisme politique des Aménophis

a. Aton, dieu unique, et Pharaon son Prophète, 48. - b. Le monothéisme atonien, d'inspiration sémito-asiatique, 50. 


\section{B. L'ÉGYPTE ET L'EMPIRE HITTITE: RIVALITÉ ET ENTENTE}

I. Déclin de la puissance Égyptienne en Orient . . . . . . 55

1. Nouvelles poussées nordiques. Les Achéens en Crète . . . 55 a. Les Aryens Achéens détruisent la puissance crétoise. Essor du monde égéen ou proto-grec, 55. - b. Rentrée en scène de l'Empire Hittite, 56.

2. Déclin de la puissance égyptienne en Orient . . . . . .

a. Les archives diplomatiques d'El-Amarna, 57. - b. Troubles et insécurité dans le Protectorat égyptien, 58. - c. Phéniciens et Amorréens aux prises. Le roi d'Amourrou (Syrie) convoite les ports libanais, 59. d. L'unité religieuse de l'empire se révèle inefficace, 60.

3. Les Hittites, maîtres de la Syrie-Nord . . . . . . . . 61

4. L'Egypte évacue les provinces orientales . . . . . . . . 63

II. REDRESSEMENT de LA PUISSANCE ÉGYPTIENNE . . . . . . . . 64

1. Reconquête des provinces orientales (Canaan et Phénicie) . . 65

a. Conquête de la Palestine. Une invasion libyenne repoussée, 65. - b. Conquête de la Phénicie. Bataille indécise de Kadesh (1315), 65.

2. Les campagnes de Ramsès II ou la grande guerre égypto-hittite (1293-1288)

3. Le jeune monde achéo-égéen, attiré par le vieil Orient . . . 68

a. Le monde égéen: pays et peuple, 68. - b. La thalassocratie achéenne. Expansion maritime, 69. - c. L'impérialisme achéen. Les Phéniciens éliminés de l'Egée, 69. - d. Expansion économique des Achéens en Phénicie, 70. - e. Les Achéens contre l'Egypte, 70.

4. Paix et entente égypto-hittites. Partage des régions syriennes .

a. La paix égypto-hittite (1278), 71. - b. Le traité de 1278, 71. - c. Partage des régions syriennes, 71. - d. Damas, Etat neutre, 72. - e. Indépendance du royaume de Gebal-Byblos, 72. - f. Ougarit, ville cosmopolite, 73. - g. L'entente égypto-hittite scellée par un mariage, 73.

5. Nouvelle marée nordique (vers 1200). Les Peuples de la Mer et du Nord

\section{LES CIVILISATIONS ORIENTALES AU IIe MILLENAIRE. CIVILISATIONS EGYPTIENNE, MESOPOTAMIENNE, HITTITE, EGEENNE, PHENICIENNE}

I. La civilisation ÉgyptienNe Au IIe Millénaire . . . . . . 77

1. L'administration . . . . . . . . . . . . . . . 77

2. L'art . . . . . . . . . . . . . . . . . . 78

3. Littérature . . . . . . . . . . . . . . . . . 79 
II. Les civilisations mésopotamiennes et anatoliennes. CiviliSATIONS KASSITE, ASSYRIENNE, MITANNO-HOURRITE, HITTITE . . 80

1. Civilisation des Kassites de Babylonie . . . . . . . . 80

2. Civilisation assyrienne . . . . . . . . . . . . 81

a. Administration, 81. - b. Le droit, 81. - c. Littérature. Religion, 82.

3. La civilisation mitanno-hourrite . . . . . . . . . . 82

a. Administration, droit, religion, art, 83.

4. La civilisation hittite .

a. Le roi, 83. - b. Organisation politique de l'Etat, 83. - La société, 84. - d. Le droit, 84. - e. Art, langue, littérature, écriture, 85. - f. Religion, 86.

III. La civilisation égéenne aU IIE. millénaire. Civilisation CRÉTOISE ET ACHÉENNE OU CRÉTO-MYCÉNIENNE . . . . . . 88

1. Origines orientales de la civilisation crétoise . . . . . . 88

2. Epanouissement de la civilisation crétoise (2000-1750) . . . 89

3. Période créto-minoenne: $1700-1400$. . . . . . . . 90

4. Période créto-achéenne ou mycénienne: $1400-1200$. . . . 92

IV. La civilisation phÉNicienNe au IIE milléNaire . . . . . 94

1. Croyances religieuses . . . . . . . . . . . . . . . . 94

2. Vie sociale et politique . . . . . . . . . . . . . 95

3. L'art phénicien, produit composite . . . . . . . . . 98

4. Vie littéraire. Les poèmes phéniciens de Ras Shamra . . . . 102 a. L'épopée du roi Kéret, 103. - b. La légende du roi Danel, 104. - c. Poème de la naissance des dieux gracieux et beaux, 104. - d. Le poème de Sib'ani, 105. - e. Valeur littéraire et morale des poèmes de Ras Shamra, 105.

V. L'INVENTION DE L'ALPHABET PHÉNICIEN . . . . . . . . . 107

1. L'alphabet, création phénicienne. Epoque de l'invention . . . 107

2. Aboutissement d'une longue suite d'expériences . . . . . 107

3. Expériences alphabétiques parallèles, contemporaines, antérieures et postérieures . . . . . . . . . . . . 108

4. Les alphabets sud-arabiques . . . . . . . . . . . 109

5. Importance et signification réelle de l'invention alphabétique . 110

6. L'alphabet phénicien, ancêtre des alphabets modernes . . . 111

7. La création du livre, initiative phénicienne. Byblos ou la «Cité du Livre». . . . . . . . . . . . . . . . . 113

8. Conclusion . . . . . . . . . . . . . . . . . 114 
SIXIEME PERIODE: $1200-750$

FLOTS NORDIQUES ET VAGUES SEMITIQUES.

INVASION DES «PEUPLES DE LA MER ET DU NORD».

MODIFICATIONS ETHNICO-LINGUISTIQUES ET MORCELLEMENT POLITIQUE

\section{A. L'INVASION DES «PEUPLES DE LA MER ET DU NORD» ET SES CONTRECOUPS DANS LE PROCHE-ORIENT}

I. Invasion des «Peuples de la Mer et du Nord» . . . . . . . . 119

1. L'Empire Hittite désorganisé par les avant-gardes de la marée . 119

2. La grande marée nordique dans l'Egée et en Asie Mineure . . 120

3. Libyens et Achéens attaquent l'Egypte par l'Ouest . . . . . 120

4. Remous en Palestine et en Syrie . . . . . . . . . . 121

5. Une nouvelle attaque libyenne repoussée par Ramsès III . . 122

6. Les Doriens envahissent la Grèce . . . . . . . . . . 122

7. Les Achéens, submergés, prennent la mer . . . . . . . 123

8. Invasion de la Syrie. Disparition de l'Empire Hittite . . . . 123

9. L'Egypte isolée se replie sur elle-même . . . . . . . . 123

10. Victoire de Ramsès III (1192) . . . . . . . . . . . . . 124

II. LES RÉGIONS MARITIMES AU LENDEMAIN DE 1200 . . . . . 125

1. Modification de la physionomie ethnique et linguistique du monde de l'époque . . . . . . . . . . . . . . 125

2. L'Egypte au lendemain de 1200: des Nordiques libyens s'installent dans le Delta . . . . . . . . . . . . . 125

3. Le côte de Canaan, royaume philistin . . . . . . . . . 126

a. Les Philistins et leur pays d'origine, 126. - b. Les Philistins, peuple évolué et belliqueux, 126.

4. Le territoire phénicien considérablement amoindri. Les Cananéens de Palestine se réfugient au Liban . . . . . . . 127

5. La Syrie-Nord, royaumes néo-hittites. Alep et Damas, principautés sémitiques . . . . . . . . . . . . . . .

a. Les Hittites de Syrie-Nord ou Néo-Hittites, 128. - b. L'influence des Néo-Hittites, 128. - c. Alep et Damas, Etats sémites indépendants. La Haute Syrie, mosaïque de races, 128. - d. Prépondérance des NéoHittites, 129.

III. LES RÉGIONS INTÉRIEURES AU LENDEMAIN DE 1200 . • . . . 130

1. Les Israélites pénètrent en «Terre Promise» . . . . . . 130

a. Epoque de la pénétration, 130. - b. Moïse, libérateur d'Israël, 130. c. Yahvé, Dieu d'Israël, 130. - d. Mö̈se, législateur et chef de nation, 131. - e. Israël aux portes de Canaan, 131. - f. Josué et la conquête, 132. 
2. Les Araméens «errants» se stabilisent en Mésopotamie, Syrie intérieure, Transjordanie . . . . . . . . . . . . 132

a. Les Araméens en Syrie. Aramisation de la région, 132. - b. Damas, centre principal des Araméens de Syrie, 134. - c. Les Araméens en Mésopotamie. Leurs luttes contre les Assyriens, 134.

3. Conclusion .

\section{B. ECLIPSE DES GRANDES PUISSANCES IMPERIALES.} MORCELLEMENT POLITIQUE DU MONDE PROCHE-ORIENTAL

I. L’Egypte, de 1200 À 750. Décadence, MORCELlement, FÉODALISME, ISOLATIONNISME . . . . . . . . . . . . . . . 139

1. Déclin de l'Egypte . . . . . . . . . . . . . . 139

a. Décadence et morcellement, 139. - b. Abandon définitif de la Palestine, 140.

2. Rôle omnipotent du grand prêtre d'Amon .

a. Accroissement du pouvoir clérical, 140 . $-\mathrm{b}$. Avènement des prêtresrois. Monarchie dualiste. La XXIe dynastie: 1085-950, 141. - c. Les oracles se substituent aux lois, 141 .

3. Les pharaons libyens. XXIIe et XXIIIe dynasties (950-730) 142

a. Les mercenaires libyens, bouclier de l'Egypte, 142. - b. Sheshonq I, fondateur de la dynastie libyenne, 143. - c. Politique extérieure de Sheshonq I. Intervention armée en Canaan, 143. - d. Politique intérieure des pharaons libyens, 145 .

II. DÉsagrégation du monde proche-ORiental. Emancipation de la Phénicie, de la Palestine et de la Syrie . . . . . 146

1. La Mésopotamie désorganisée par les Araméens. Babylonie, Assyrie, Elam luttent pour l'hégémonie . . . . . . . . 146

a. Les Elamites, maîtres de Babylone (1175), 146. - b. La dynastie élamite de Babylone se détache de l'Elam, 146. - c. Les nomades araméens infestent la contrée, 147. - d. Araméens autochtones, puis Assyriens, maîtres de Babylone (1095-900), 147.

2. L'Asie Mineure ruinée et morcelée. Les Phrygiens recouvrent les Hittites détruits . . . . . . . . . . . . . . 148

3. Eclipse des grandes puissances impériales. Emancipation de la Phénicie, de la Palestine et de la Syrie. Morcellement, divisions et conflits . . . . . . . . . . . . . . . 148

C. LA PHENICIE INDEPENDANTE: 1200-750. L'EMPIRE PHENICIEN, PREMIER EMPIRE COLONIAL, MARITIME ET COMMERCIAL

I. La Phénicie libanaise ou métropolitaine . . . . . . . 153

1. Au lendemain du cataclysme de 1200 . . . . . . . . . . 153 
2. Les cités-Etats de Phénicie-Liban: Arvad, Gebal, Sidon, Tyr . 154

a. L'Etat d'Arvad (Rouad), 154. - b. L'Etat de Gebal (Byblos), 154. -

c. L'Etat de Sidon (Saïda), 155. - d. L'Etat de Sour (Tyr), 155.

3. La Phénicie, ligue d'Etats autonomes. Tripoli, siège de la ligue . 156

II. Expansion des Phéniciens vers l'Occident et fondation de LEUR EMPIRE MARITIME

1. La thalassocratie phénicienne . . . . . . . . . . . 158

a. Expansion des Phéniciens vers l'Occident, 158. - b. Période de fondation de l'empire: 1200-1000, 159.

2. L'empire phénicien, premier empire maritime et commercial . 160

a. Domaine de l'empire, 160. - b. Naissance à la civilisation d'un nouveau monde, 160. - c. Méditerranée orientale et Mer Egée, 160. - d. Méditerranée centrale et occidentale, 161. - e. Côtes de l'Atlantique, 161. - f. Espagne atlantique, 161. - g. Comptoirs et concessions, 162.

- h. Caractère original de l'empire phénicien, 162.

3. La Phénicie, centre de gravité économique mondial .

a. La Phénicie, centre économique mondial, 163. - b. La Phénicie, puissance commerciale, 164. - c. La Phénicie, puissance financière et politique, 166 .

III. La Phénicie et les pays voisins, DE 1200 À 750. RapPorts avec la Palestine et Isrä̈L.

1. Expansion économique dans les pays continentaux .

a. Les Phéniciens et l'Arabie, 167. - b. Les Phéniciens et la Palestine, 167.

2. La Phénicie, de 1200 à 900

a. De 1200 à 1000: période obscure, 168. - b. La Phénicie, vers l'an 1000, 169. - c. Abibaal, roi de Tyr (980-969), allié du roi David, 169. - d. Hiram I et Salomon, amis et alliés, 170. - e. Condominium phénico-salomonien sur la Palestine, 170. - f. Déclin de la dynastie à Tyr et à Jérusalem. Agitations sociales et révolutions politiques, 172.

3. La Phénicie, de 900 à 750: rapports avec Samarie, Jérusalem et Damas

a. Alliance de Tyr et de Samarie, 173. - b. Rétablissement de l'influence phénicienne à Samarie et à Jérusaiem, 174. - c. Jézabel à Samarie, 174. - d. Entente d'Israël et de Juda. Athalie à Jérusalem, 174. - e. L'influence phénicienne éliminée de Palestine. Massacre de Jézabel et d'Athalie, 175. - $\mathrm{f}$. Révolution à Tyr, triomphe de la faction démocratique, 175. - g. Fondation de Carthage en Afrique, 175. - h. Déclin de Tyr en Occident. Essor de Carthage, 176. - i. Nuages assyriens à l'horizon, 176.

D. PALESTINE ET SYRIE INTÉRIEURE, DE 1200 À 750

I. LA MONARChIE UNITAIRE D'ISRAËL. JÉRUSALEM ET TYR, ALliÉES

ET ASSOCIÉES 
1. Les Isrälites en Canaan. Saül (1025-1010), premier roi d'Israel 181

a. L'anarchie en Israël; prépondérance des Philistins, 181. - b. Le pouvoir des Suffètes ou Juges, 182. - c. Le juge Samuel et la fondation de la monarchie, 182. - d. Saül, premier roi d'Israël, 183.

2. Le roi David (1010-955), créateur de l'Etat national .

a. Etendue du royaume de David, 183. - b. Yahvé, Dieu unique et universel. David, prophète et prêtre de Yahvé, 184. - c. Yahvé, Dieu intolérant et jaloux, 185. - d. Mort de David, 185.

3. Le roi Salomon (955-933). Apogée de la monarchie .

a. Opulence et faste de Salomon, 186. - b. Déclin de la monarchie; mort de Salomon, 186.

4. Le royaume de Salomon, zone économique d'influence phénicienne

a. Les Phéniciens dirigent l'économie israélite, 187. - b. Le Temple de Jérusalem, œuvre phénicienne, 188. - c. Prépondérance phénicienne à Jérusalem, 188. - d. Affinités ethniques et culturelles, 188. - e. Réaction antiphénicienne, 189.

II. Les royaumes d'IsRaËl, de Juda et de Damas. Rivalités et CONFLITS

1. Les royaumes d'Israël et de Juda

a. Démembrement du royaume de Salomon, 190 . - b. Les deux royaumes d'Israël et de Juda. Scission politique et religieuse, 190. - c. Décadence politique du peuple élu, 191.

2. Israël, Juda, Damas: rivalités et entente .

a. Omri, roi d'Israël, 192 - b. Alliance de Samarie et de Tyr. Retour de l'influence phénicienne, 192. - c. Achab, vainqueur de Damas, 192. d. Alliance d'Achab et de Juda. L'influence phénicienne à Jérusalem, 192. - e. Mort d'Achab et déclin de sa dynastie, 193.

3. Réaction nationale et religieuse à Samarie et à Jérusalem .

a. Meurtre de Jézabel (846) et d'Athalie (841), 193. - b. Les luttes des roitelets syro-palestiniens attirent l'Assyrien du Nord, 194. - c. Le royaume de Damas, province assyrienne $(733), 195 .-\mathrm{d}$. Le royaume de Samarie, province assyrienne. Celui de Jérusalem, vassal et tributaire (722), 195. - e. Le peuple élu châtié par Yahvé, 195.

E. LE PROCHE-ORIENT DES PLATEAUX DU NORD (1200-750)

I. Assyrie, Arménie, Iran occidental . . . . . . . . . 199

1. Le royaume d'Assyrie, de 1200 à 900 . . . . . . . . . . 199

2. Les Araméens menacent la Mésopotamie . . . . . . . 200

3. Le royaume d'Ourartou, en Arménie . . . . . . . . . 201

4. Eveil de l'Iran occidental; apparition des Mèdes et des Perses . 202

a. Eveil de l'Iran à la civilisation, 203. - b. Le fer et les chevaux attirent les Assyriens en Iran, 203. - c. Stabilisation des Mèdes, Perses et Parthes, 204. 
II. L'ASSYRIE ET LES ROITELETS SYRO-PALESTINIENS . . . . . 205

1. Ascension de l'Assyrie . . . . . . . . . . . . . 205

a. Bataille de Qarqar, victoire indécise (854), 205. - b. Salmanasar III défait une seconde coalition syrienne, 206. - c. Salmanasar III, suzerain de Babylone, 206. - d. L'Assyrie, maîtresse du pays des Deux-Fleuves, 206. - e. Les Assyriens pillent Damas (805), 207. - f. Eclipse de l'Assyrie (800-740), 207. - g. Fondation de l'Empire assyrien, 207.

2. Elaboration du futur monde gréco-égéen . . . . . . . 208

3. Cilicie, Chypre . . . . . . . . . . . . . . . . 209

SEPTIËME PERIODE: 750-540

VAGUES NORDIQUES: LES CIMMERIENS ET LES SCYTHES.

RENAISSANCE DE L'IMPERIALISME MESOPOTAMIEN.

EXPANSION DES ASSYRIENS, OURARTEEENS, CHALDEENS, MEDES, LYDIENS

A. LE GRAND EMPIRE ASSYRIEN. FONDATION, APOGEE, DESTRUCTION (740-612). L'ASSYRIE CONTINENTALE ATTIREE PAR LA MER

I. L'ASSYRIE, JUSQU'À LA FONDATION DU GRAND EMPIRE . . . . . . 215

1. L'impérialisme assyrien . . . . . . . . . . . . . 215

a. L'Assyrie, Prusse de l'Orient ancien, 215. - b. Le pays assyrien, 215.

- c. Composition ethnique, 216. - d. L'impérialisme assyrien, 217.

2. Aperçu récapitulatif jusqu'à 750 . . . . . . . . . . 218

II. L'EMPIRe ASSYRIEn ET L'EGYPTe, de 722 À 689 . . . . . . 220

1. Le cadre historique vers 750 . . . . . . . . . . . 220

2. Nouvelles vagues nordiques en Asie Mineure. Expansion, vers le Sud, des Cimmériens et des Scythes . . . . . . . . 221

a. Cimmériens au nord de la Mer Noire. Scythes au nord de la Caspienne, 221. - b. Nomades cavaliers et guerriers redoutables, 221. - c. Les Cimmériens en Asie Mineure, 222. - d. Les Scythes dans le nord de l'Iran, 222.

3. Téglatphalasar III (745-727), maître du Croissant Fertile et de l'Arménie . . . . . . . . . . . . . . . . .

a. Conquête de la Syrie et de la Palestine (738-732), 223. - b. Prise de Babylone (731), 224. - c. Mort de Téglatphalasar et révolte de la Phénicie, 224.

4. Au temps de Sargon II (722-705)

a. Consolidation des conquêtes. Destruction du royaume d'Israël (722), 224. - b. Révolte en Syrie. Bataille de Qarqar (720), 225. - c. Babylone reprise sur les Chaldéens (710), 225. - d. Pacification de l'Arménie et de l'Asie Mineure. Prise de Chypre (709), 225. - e. Mort de Sargon II (705), 226. - f. L'œuvre de Sargon II, 226. 
5. Sargon II et l'Egypte . . . . . . . . . . . . . . . 226

a. Impuissance de l'Egypte. Invasion éthiopienne (730), 226. - b. Les Egyptiens battus près de Gaza (720), 227.

6. Au temps de Sennachérib (705-681) . . . . . . . . . 228

a. Consolidation des conquêtes, 228 . - b. Soulèvement et défaite des Chaldéens de Babylone (704), 228. - c. Soulèvement et soumission des Phéniciens (701), 228. - d. Reconquête de la Palestine (701), 229. - e. Révolte de Babylone (700), 229.

7. Sennachérib et l'Egypte .

a. Les Ethiopiens reconquièrent l'Egypte, 229. - b. Intrigues de Pharaon en Palestine, 229. - c. L'Egypte échappe à l'invasion, 230. - d. Le pharaon Taharka rétablit l'unité monarchique, 230. - e. Mort de Sennachérib (681), 230.

III. L'EMPIRE ASSYRIEN ET LA CONQUÊTE DE L'EgYPTE. DéCliN ET RUINE DE L'EMPIRE

1. L'Empire assyrien au temps d'Asaraddon (689-669). Première conquête de l'Egypte .

a. Pacification de la Babylonie, 231. - b. Révolte et répression de Sidon (676), 231. - c. Asaraddon en Arabie, 231. - d. Tyr, assiégée, résiste victorieusement, 232. - e. Première conquête de l'Egypte (671), 232. - f. Révolte de l'Egypte (669), 233.

2. L'Empire assyrien au temps d'Assourbanipal (669-626). Apogée et déclin . . . . . . . . . . . . . . . a. L'Egypte, reconquise et reperdue (666- 663), 233. - b. Répression des révoltes, 235. - c. Déclin et ruine de l'Empire assyrien, 236.

IV. LA CIVILISATION ASSYRIENNE . . . . . . . . . . . . . . . 239

1. Considérations sur la précarité de l'Empire assyrien . . . . 239

a. Antagonisme de la terre (Assyrie) et de la mer (Phénicie, Egypte), 239.

- b. Rôle historique de l'Empire assyrien, 240.

2. Civilisation .

a. Le roi, l'armée et la marine, 241. - b. La société assyrienne, 242. c. Le port du voile pour les femmes, 242 . - d. Le droit, 242. - e. La religion, 243. - f. Littérature et science, 243. - g. La médecine, 243. h. L'art assyrien, 244.

B. L'EMPIRE ASSYRIEN DEMEMBRE; LE PROCHE-ORIENT MORCELE. EGYPTE, CHALDEE, MEDIE, SE DISPUTENT L'HÉGEMONIE (612-540).

I. La CARTE POlitique après LA RUINe DE L'AssYrie. Les GRANDS Etats successeurs: Egypte, Chaldée, Médie, Lydie . . . 247

1. L'Egypte, sous la XXVIe dynastie saïte (663-525), se tourne vers la mer. Le Delta s'ouvre aux mercenaires et commerçants grecs .

a. Avènement de la XXVIe dynastie, 247. - b. Psammétique I (663609), unificateur et libérateur de l'Egypte, 247. - c. Psammétique en 
Palestine, 248. - d. La Palestine, vieille zone de protection, est occupée par l'Egypte (609), 248. - e. Les mercenaires grecs remplacent les Libyens, 249. - f. Les colonies grecques marchandes, 250. - g. La citéport hellénique de Naucratis, 250. - h. Rapports des Egyptiens et des Grecs, 250. - i. L’Egypte saïte, puissance méditerranéenne. Le Delta, centre politique de la monarchie, 250. - j. Expansion maritime vers le Sud, 251. - k. Amélioration de la condition des classes populaires, 251. - 1. Prospérité de l'Egypte. Renaissance des arts, 252.

2. La Chaldée ou Néo-Babylonie. Babylone sous le règne de Nabuchodonosor II (605-562) . . . . . . . . . .

a. La Chaldée jusqu'à Nabuchodonosor II, 252. - b. Babylone et Nabuchodonosor, 254. - c. Babylone, 254. - d. La cité et le palais royal, 255. - e. Le fameux Esagil ou temple de Mardouk, 225. - f. Les jardins suspendus, 256. - g. La Tour de Babel, 256. - h. Les murs de Babylone, 257. - i. La civilisation chaldéenne ou néo-babylonienne, 258.

3. La Médie, en Iran occidental, jusqu'à 612 . . . . . . . 259

a. Mèdes et Perses vers 700,259 . - b. Naissance de la monarchie mède, 259. - c. La Médie vassale des Scythes (653-625), 260. - d. La Médie émancipée triomphe de l'Assyrie, 260. - e. L'Empire des Mèdes, 260.

4. La Lydie et sa capitale Sardes, jusqu'à 615 . . . . . . . . 261

a. Le roi Gygès (687--652), 261. - b. Rôle historique de la Lydie, 262.

II. Rapports des Egyptiens, Chaldéens, Mèdes, Lydiens. Course À L'hégémonie politique du Proche-Ortent . . . . . . . 263

1. L'équilibre oriental au lendemain de 612 . . . . . . . . 263

2. Egyptiens et Chaldéens convoitent la Palestine . . . . . . 263

3. Mèdes et Lydiens convoitent l'Anatolie . . . . . . . . 264

4. Le royaume de Juda, jusqu'à 609 . . . . . . . . . . . . 264

5. Défaite et mort de Josias, roi de Juda (609) . . . . . . 265

6. Les Egyptiens s'annexent Palestine et Syrie (609) . . . . . . 265

7. Les Egyptiens vaincus et expulsés (605) . . . . . . . . 265

8. L'équilibre international . . . . . . . . . . . . . 266

9. Révoltes de Tyr et de Jérusalem contre Babylone . . . . . 266

a. Tyr, tête de la révolte (597), 266. - b. Défaite et mort de Yoakim, roi de Juda. Le premier Exil (597), 267.

10. Politique maritime et africaine de l'Egypte .

11. Nouvelle révolte et destruction du royaume de Jérusalem. La dispersion du peuple juif (586) . . . . . . . . . . . 268

12. Soumission de $\operatorname{Tyr}(573)$. . . . . . . . . . . . . . . . 269

13. Insuccès égyptiens en Cyrénaïque (568) . . . . . . . . 269

14. Amasis, pharaon pacifique (568-525) . . . . . . . 270

15. La république à $\operatorname{Tyr}(564)$. . . . . . . . . . . . 270 
16. Chaldée, Médie, Lydie et Grecs (612-539) . . . . . . 270

a. Fortification de Babylone, 270. - b. Lydiens et Mèdes, rivalité et entente (591-585), 271. - c. Les Arméniens en Ourartou (vers 600), 271. - d. Prospérité de la Lydie sous Crésus (561-546), 271. - e. Astyage, dernier roi de race mède (584-555), 272.

17. Chute de la dynastie mède en Iran. Avènement de la dynastie des Perses achéménides (555) . . . . . . . . . . . 272

a. La dynastie royale des Perses achéménides (555-330), 272. - b. Cyrus le Perse (555-528) se substitue à Astyage, 272. - c. La Médie devient la Perse, 273. - d. Cyrus organise son nouveau royaume, 274. e. Décadence de la dynastie babylonienne, 274. - f. Nabonide à Hama et à Tyr (553-552), 274. - g. Inquiétude en Egypte. Alliance avec Crésus et Nabonide, 274.

III. LA RELIGION IRANIENNE SOUS LES ROIS MÈDES. LA RÉFORME RELIGIEUSE DE ZOROASTRE . . . . . . . . . . . . 276

1. Religions des Iraniens . . . . . . . . . . . . . 276

2. La réforme religieuse de Zoroastre ou le mazdéisme zoroastrien $\mathbf{2 7 7}$

a. Zoroastre, réformateur et fondateur de religion, 277. - b. Aspect religieux de la réforme de Zoroastre, 277. - c. Immortalité de l'âme. Jugement après la mort, 278. - d. Le culte zoroastrien, 279. - e. Valeur morale de la doctrine de Zoroastre, 279. - $\mathrm{f}$. Aspect social de la réforme de Zoroastre, 280. - g. Zoroastre, Bouddha, Confucius, presque contemporains, 281. - h. Rôle du zoroastrisme dans l'Etat, 282.

HUITIEME PERIODE: 540-330

ORGANISATION ET EXPANSION DU MONDE IRANIEN. L'EMPIRE PERSE, PREMIER GRAND EMPIRE

PROCHE-ORIENTAL. LE PROCHE-ORIENT ATTIRE PAR LA MER

\author{
A. LE GRAND EMPIRE PERSE. \\ FONDATION, CONSOLIDATION, ORGANISATION
}

I. Fondation de l'Empire. Cyrus et Cambyse . . . . . . . 289

1. L'Empire perse achéménide. Premier grand Empire indoeuropéen . . . . . . . . . . . . . . . . . 289

2. Caractère de la domination perse . . . . . . . . . . 290

3. Cyrus le Grand, fondateur de l'Empire . . . . . . . . 291

4. Caractère des Perses . . . . . . . . . . . . . . 292

5. Les conquêtes de Cyrus . . . . . . . . . . . . . 292

a. Conquête de l'Asie Mineure (546), 292. - b. La Lydie, satrapie 
perse, 293. - c. Conquête de l'Est jusqu'à l'Indus (545-539), 293. d. Prise de Babylone (539), 294. - e. La Phénicie accueille Cyrus comme un libérateur, 294. - f. Libération des Juifs (538), 295. - g. Mort de Cyrus (528), 295.

6. Cambyse et la conquête de l'Egypte .

a. Préparatifs, 295. - b. Conquête de l'Egypte (525), 296. - c. L'Egypte, satrapie perse, 296. - d. Projet de conquérir Carthage, 297. - e. Expédition contre l'Oasis de Siouah, 297. - f. Expédition contre l'Ethiopie, 297. - g. Comportement de Cambyse en Egypte, 298. - h. Révolte en Perse (521), 298. - i. Mort de Cambyse (521), 298. - j. Soulèvement des provinces conquises, 298. - k. Avènement de Darius I, 298.

II. Restauration et organisation de L'Empire . . . . . . . 300

1. Darius I, restaurateur de l'Empire . . . . . . . . . 300

a. Pacification des révoltes, 300. - b. Darius en Egypte (517), 300. - c. Essor économique de l'Empire reconstruit, 301.

2. Organisation et administration de l'Empire .

a. Politique de Darius, 302. - b. Création et organisation interne des satrapies, 302. - c. Le tribut des provinces, 303. - d. Les métropoles de l'Empire, 303. - e. Routes impériales, 304. - f. Etalon monétaire, 304. - g. L'armée et la flotte, 305.

3. Langues et écritures des Perses. L'araméen, langue officielle de l'Empire

4. Caractère divin de la royauté. Le roi, représentant du grand Justicier céleste

5. La satrapie d'Abarnahara: Phénicie, Palestine, Syrie, Chypre . 306

a. Subdivisions de la satrapie d'Abarnahara, 307. - b. Le territoire d'Arabaya ou Arubu (Arabes), 307. - c. La Phénicie sous la domination perse, 308. - d. Emancipation des colonies phéniciennes d'Occident, 309. - e. Rapports de la Phénicie avec la Perse, 309.

\section{B. L'EMPIRE PERSE ET LE MONDE GRECO-ÉGÉEN}

I. Les Guerres MédiQues ou gréco-Perses . . . . . . . . . 313

1. Origines des Guerres Médiques . . . . . . . . . . 313

a. Vers 1'Empire universel, 313. - b. Darius convoite le monde égéen, 313. - Politique maritime de Darius, 314. - d. Les Grecs, en face des Perses, 314. - e. Campagne perse contre les Scythes de Russie, 315. f. Préparatifs de guerre. Soumission de Byzance (513), 315. - g. Occupation de la Thrace; soumission de la Macédoine (512), 316. - h. Bilan de la campagne contre les Scythes, 316. - i. Révolte des cités grecques d'Asie Mineure (498), 316. - j. Défaite des Grecs d'Asie (496), 317. k. Les Guerres Médiques, duel entre deux mondes, 317.

2. Les Guerres Médiques (492-466) . . . . . . . . . 318

a. Reconquête de la Thrace et de la Macédoine (492), 318. - b. Bataille de Marathon (490), 318. - c. Révolte de l'Egypte et mort de Darius (485), 319. - d. Impuissance du colosse perse, 319. - e. Xerxès, prince 
faible et violent, 320. - f. La révolte de l'Egypte sauvagement réprimée, 320. - g. Babylone, révoltée, est prise et détruite (482), 320. - h. Préparatifs de guerre contre la Grèce, 321. - i. Forces terrestres et navales, 321. - j. Alliance avec Carthage, 321. - k. La grande armée traverse les Détroits, 322. - 1. Bataille des Thermophyles et prise d'Athènes (480), 322. - m. Défaite navale des Perses à Salamine (480), 322. - n. L'amiral phénicien exécuté par Xerxès, 323. - o. Les Perses battus et rejetés en Asie (466), 323. - p. Signification morale de la victoire grecque, 323. - q. Athènes, maîtresse du monde égéen, 324. - r. L'Empire perse ébranlé et affaibli, 324.

II. Décadence et ruine de l'Empire perse . . . . . . . . 326

1. Rapports des Perses et des Grecs . . . . . . . . . . . 326

a. Révoltes en Bactriane et en Egypte, 326. - b. Traité de paix grécoperse (449), 326. - c. Renaissance de la nation juive en Palestine (445), 326. - d. Essor de la civilisation hellénique et son rayonnement dans le Proche-Orient, 327. - e. Intrigues d'Athènes en Asie Mineure, 328, f. Révolte de Cyrus le Jeune (401). La Retraite des Dix Mille, 328. - g. Emancipation de l'Egypte (404), 329. - h. La paix gréco-perse d'Antalcidas (387). Récupération des villes grecques d'Asie, 329. - i. Désagrégation de l'Empire, 329. - j. L'Empire provisoirement sauvé, 330.

2. L'Egypte indépéndante (404-345) s'appuie sur les Grecs . . 330

3. Redressement et fin de l'Empire perse. Artaxerxès III, prince féroce et volontaire . . . . . . . . . . . . . . 332

a. La Phénicie révoltée est sauvagement réduite (347), 332. - b. Reconquête de l'Egypte (345), 333. - c. Résignation et décadence de l'Egypte, 333. - d. L'Empire redressé, colosse aux pieds d'argile, 334. - e. Destruction de l'Empire perse, 335.

\section{CIVILISATION ET RELIGION DES PERSES ACHÉMÉNIDES}

I. LA CIVILISATION PERSE ACHÉMÉNIDE . . . . . . . . . . . . 337

1. Le legs des rois achéménides . . . . . . . . . . . 337

2. Le mazdéisme achéménide, religion officielle . . . . . . 338

a. Les religions de la Perse, 338. - b. Le mazdéisme achéménide, religion officielle, 339. - c. Aspect religieux et politique du mazdéisme achéménide, 339. - d. Les mages et les sacrifices, 340. - e. Symboles divins et lieux de culte, 340 . - f. Aspect social de la religion achéménide. Solidarité sociale et sentiment national, 340. - g. Valeur morale de la religion achéménide, 341 .

3. La religion judaïque sous les Perses . . . . . . . . . 342

Achèvement du Temple. Refonte du Pentateuque 342. 
NEUVIEME PERIODE: 330-64 AV. J.-C.

UNIFICATION ET EXPANSION DU MONDE GRECO-EGEEN. L'EMPIRE D'ALEXANDRE. LES MONARCHIES HELLENISTIQUES

\section{A. L'EMPIRE GRECO-ORIENTAL D'ALEXANDRE LE GRAND}

I. LA GRèce et L'HeLlÉNiSMe, JUSQU'À L'EXPANSION D'AleXANDRE. ESQUISSE HISTORIQUE .

1. Le monde gréco-égéen: pays et race

a. Le pays gréco-égéen, 349 . - b. Les conditions géographiques du pays gréco-égéen et leur action sur les habitants, 349. - c. Le nom et la race, 350 .

2. La Grèce et la civilisation hellénique

a. L'hellénisme, 350 . - b. Importance de l'histoire grecque, 351. - c. Périodes de l'histoire grecque, 351.

3. La Grèce archaïque, jusqu'à 460 .

a. L'empire égéen de la Crète, 352. - b. L'empire achéo-égéen. Les Achéens, première famille grecque, 352 . - c. Les Doriens, seconde famille grecque, 352. - d. Colonisation des côtes d'Asie Mineure, 352. e. Essor des cités helléniques d'Asie Mineure, 353. - f. Réveil d'Athènes et de Sparte, 353. - g. Influence de la culture orientale, 353.

4. La Grèce classique: Ve et IVe siècles. Apogée de la civilisation hellénique

a. Athènes, «école de la Grèce», 354. - b. L'hellénisme et la culture orientale, 354. - c. Rivalité d'Athènes et de Sparte, 355.

5. La Grèce macédonienne . . . . . . . . . . . . . 355

a. La Macédoine, 355. - b. Philippe II de Macédoine, unificateur de la Grèce, 356. - c. Expédition manquée contre Byzance, 356. - d. La Grèce sous le joug macédonien, 356. - e. Alexandre, roi de Macédoine, 357.

6. Hellénisme et orientalisme avant Alexandre . . . . . . 357

7 L'Orient antique, école de la Grèce . . . . . . . . . 357

II. LeS CONQUÊtes et L'EMPIRE D'AleXANDRE . . . . . . . . 359

1. Invasion de l'Asie Mineure . . . . . . . . . . . . . . . 359

a. Sur le site de Troie (Ilion), 359. - b. Bataille du Granique (333), 360.

2. Bataille d'Issus (332). Conquête de la Syrie-Nord . . . . 361

3. Soumission de la Phénicie. Siège et destruction de Tyr . . . 361

a. Importance de la Phénicie pour Alexandre, 361. - b. Soumission de Marathos, Arvad, Gebal, Sidon, 363. - c. Siège de Tyr, 364. - d. Prise et destruction de Tyr (332), 365. - e. Disparition de Tyr comme puissance maritime, 366. - f. Propositions perses rejetées par Alexandre, 366.

4. Prise de Gaza et reddition de Jérusalem . . . . . . . . . . 367

a. Siège et prise de Gaza, 367. - b. Reddition de Jérusalem, 367. 
5. Soumission de l'Egypte .

a. Alexandre accueilli en libérateur, 368. - b. Alexandre, pharaon d'Egypte (332), 368. - c. Fondation d'Alexandrie, 369. - d. Pèlerinage à l'oasis de Siouah, 369.

6. Conquête de la Mésopotamie, de la Perse et de l'Asie .

a. Défaite de Darius près de Ninive (331), 370. - b. Alexandre, roi de Babylone et d'Asie, 370. - c. Soumission de la Susiane, 370. - d. Faste des officiers gréco-macédoniens, 370. - e. Projets de conquêtes vers l'Est, 371. - f. Soumission de l'Iran, 371. - g. Conquête de l'Inde, 372. - h. Sur les côtes de l'Océan Indien, 373. - i. Retour d'Alexandre à Suse (324), 373. - j. Mariages gréco-perses, 374.

7. Mort d'Alexandre (323) . . . . . . . . . . . . . 374

III. Alexandre le Grand et son oeuvre. Désagrégation et PARTAGE DE SON EMPIRE . . . . . . . . . . . . . . . . 376

1. Le personnalité d'Alexandre . . . . . . . . . . . 376

a. Missionnaire et héros, 376. - b. Chef et organisateur, 377. - c. Un mystique convaincu, 377.

2. L'œuvre d'Alexandre le Grand. Unité culturelle du monde proche-oriental . . . . . . . . . . . . . . . 378

a. Annexion matérielle et morale, 379. - b. Economie et civilisation universelles, 379. - c. Fusion de l'Orient et de l'Occident, 380.

3. L'Empire d'Alexandre, jusqu'au partage définitif. Aperçu historique: 323-275 .

a. La succession d'Alexandre. Le général Perdiccas, régent de l'Empire, 381. - b. Les Gouverneurs des provinces, 381. - c. Une révolte d'Athènes est écrasée (322), 381. d. Rivalités entre les Gouverneurs des provinces. Meurtre de Perdiccas, 382. - e. Régence d'Antipatros (321-319), 382. - f. Régence de Polyperchon. Révolte de Cassandre, 382. - g. Antigone, maître de l'Asie, 382. - h. Antigone et son fils Démétrius, maîtres de la Grèce, 383. - i. Bataille d'Ipsos. Défaite et mort d'Antigone (301), 383. - j. Défaite et mort de Démétrius (283), 384. - k. Défaite et mort de Lysimaque (281), 384. - I. Séleucus, maître de l'Asie, disparaît en 280, 384. - m. Antigone Gonatas, maître de la Grèce, 384. - n. Démembrement et partage définitif de l'Empire d'Alexandre (275), 385. - o. Caractère éphémère de l'œuvre politique d'Alexandre, 385.

\section{B. LES ROYAUTES HELLÉNISTIQUES DU PROCHE-ORIENT:} L'ASIE, L'EGYPTE

\section{L'Empire gréco-asiatique des Séleucides (Haute Syrie-} MÉsopotamie) . . . . . . . . . . . . . . . . 389

1. Formation et étendue de l'Empire séleucide . . . . . . . . . 389

a. Politique impériale de Séleucus I (312-280), 389. - b. Antioche et Séleucie du Tigre, capitales impériales, 389. - c. Causes des futurs conflits égypto-asiatiques, 389. 
2. La Syrie séleucide ou Haute Syrie . . . . . . . . . 390

3. Babylonie, Iran et Asie Mineure . . . . . . . . . 392

a. La Babylonie séleucide, 392. - b. L'Iran séleucide, 392. - c. L'Asie Mineure, 392.

4. Phénicie .

a. Sous Alexandre, 393. - b. Sous les successeurs d'Alexandre, 394. c. Régime politique des cités phéniciennes, 394. - d. La Phénicie et la route des Indes, 395. - e. La Phénicie et l'hellénisme, 395.

II. Aperçu historique de L'Empire séleucide (275-64). Rapports aVec l'Egypte et RoMe.

1. Les Séleucides et les Lagides: rivalités et conflits. Les «guerres syriennes» ou syro-égyptiennes (275-198)

a. Causes de conflits, 397. - b. Première guerre syrienne (275-271), 398. - c. Deuxième guerre de Syrie $(261-247), 399$. - d. Troisième guerre de Syrie (245-241), 399. - e. Antiochus le Grand restaure l'Empire, 400. - f. Quatrième guerre syrienne (219-217), 400. - g. Cinquième guerre syrienne (200-198). Conquête de la Phénicie et de la Palestine par Antiochus III, 400. - h. La Grande Syrie séleucide, 401.

2. L'Empire séleucide et la puissance romaine. Rivalité et conflit armé. Défaite d'Antiochus III .

a. Germes de conflit, 401. - b. Antiochus en Thrace et à Byzance (195), 402. - c. Défaite d'Antiochus à Korykos et en Magnésie (190), 402. - d. La paix d'Apamée (188), 403. - e. Rome et l'Empire séleucide après Apamée, 403.

3. Désagrégation et ruine de l'Empire séleucide. Révolte des Juifs et querelles dynastiques .

a. Déclin de l'Empire séleucide, 404. - b. Révolte des Juifs (164), 404. - c. Querelles dynastiques, 406. - d. Indépendance de la Judée (142), 406. - e. Les Parthes occupent la Mésopotamie (129), 406. f. Extension du royaume de Judée, 407. - g. Agonie de l'Empire séleucide (120-83), 407. - h. Fin du royaume gréco-syrien des Séleucides (83), 407. - i. La Syrie, province romaine (64), 407. - j. L'œuvre politique des rois séleucides, 407 .

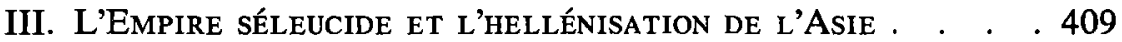

1. Les civilisations hellénistiques en général . . . . . . . 409

a. Diffusion de la civilisation hellénistique en Orient, 409. - b. La cité, fondement de l'Etat hellénistique, 409. - c. Caractère de la cité hellénistique, 409. - d. Milieu social, économique et culturel, 410. - e. Vie intellectuelle, 411. - f. Parallèle avec la société moderne, 411. - g. Régime politique des royautés hellénistiques, 412.

2. La royauté séleucide . . . . . . . . . . . . . . 413

a. Monarchie absolue de caractère divin, 413 . - b. Armée, flotte, administration, 413. - c. Les cités séleucides, 413. 
3. L'hellénisation de l'Asie . . . . . . . . . . . . . 415

a. Rôle de l'Empire séleucide, 415. - b. L'hellénisation des cités, 415. - c. Les masses populaires imperméables à l'hellénisme, 416. - d. Renaissance de la culture orientale, 416. - e. Euvre culturelle des Séleucides, 417.

IV. Le Royaume GRéCo-Égyptien des Ptolémées . . . . . . 418

1. L'Egypte sous les Ptolémées . . . . . . . . . . . 418

a. Le roi lagide, 418. - b. Administration, 419. - c. Alexandrie, seconde Athènes, 419. - d. Politique extérieure, 420. - e. l'Egypte indigène réfractaire à l'hellénisation, 420. - f. Renaissance du sentiment national égyptien, 421.

2. Aperçu historique de l'Egypte ptolémaïque .

a. Ptolémée I (322-284), fondateur de la dynastie lagide, 421. - b. Ptolémée I occupe la Syrie-Sud (301), 422. - c. Première guerre syrienne ou syro-égyptienne (275-271), 422. - d. Deuxième guerre syrienne (261-247), 423. - e. Troisième guerre syrienne (245-241), 423. - f. Quatrième guerre syrienne (219-217), 423. - g. Cinquième guerre syrienne (200-198), 424. - h. Querelles dynastiques et interventions étrangères, 424. - i. Agonie et fin de la monarchie des Lagides (116-30), 425.

3. Conclusion . . . . . . . . . . . . . . . . . 425

Planches I-V 
